Annuaire suisse de politique de développement

$19 \mid 2000$

Pour une politique plus cohérente envers les pays en développement

\title{
Crise de la dette en Tanzanie et cohérence de la politique de développement suisse
}

\section{Christine Eberlein}

\section{(2) OpenEdition}

\section{Journals}

Édition électronique

URL : http://journals.openedition.org/aspd/838

DOI : 10.4000/aspd.838

ISSN : 1663-9669

Éditeur

Institut de hautes études internationales et du développement

Édition imprimée

Date de publication : 1 avril 2000

Pagination : 73-87

ISSN : 1660-5934

Référence électronique

Christine Eberlein, «Crise de la dette en Tanzanie et cohérence de la politique de développement suisse ", Annuaire suisse de politique de développement [En ligne], 19 | 2000, mis en ligne le 18 août 2012, consulté le 08 septembre 2020. URL : http://journals.openedition.org/aspd/838 ; DOI : https:// doi.org/10.4000/aspd.838 


\section{CRISE DE LA DETTE EN TANZANIE ET COHÉRENCE DE LA POLITIQUE DE DÉVELOPPEMENT SUISSE}

Christine Eberlein*

\section{INTRODUCTION}

Alors que nous entrons dans le nouveau millénaire, l'endettement des pays les plus pauvres ne cesse de croitre. Or, non seulement ce fardeau entrave la croissance économique et l'accès à la prospérité, mais il grève aussi les structures sociales et menace la satisfaction des besoins les plus élémentaires.

La majeure partie des dettes a été accumulée dans les années 70 et 80 . On pensait alors que les flux monétaires dirigés du Nord vers le Sud favoriseraient l'essor économique des pays en développement. La politique économique internationale menée à cette époque s'est toutefois révélée contre-productive, car les pays du Sud n'ont pas été en mesure de rembourser ces apports financiers massifs et en subissent à présent le contrecoup: au lieu de pouvoir investir dans leur développement social, ils sont obligés de consacrer leurs rares devises au remboursement d'obligations contractées à l'étranger.

Aujourd'hui, tous les spécialistes admettent que l'apport de capitaux et la promotion des exportations ne suffisent pas pour garantir un développement durable. Pour garantir un développement à long terme tout en évitant les crises de la dette, il faut en effet améliorer toute une série de paramètres nationaux. Il importe ainsi d'atténuer la pauvreté et de favoriser la mise en place de structures économiques et sociales durables, de surmonter les problèmes démographiques et de préserver les ressources naturelles.

Les donateurs internationaux soutiennent les efforts des pays les plus pauvres pour se développer et pour lutter contre la pauvreté. Ce soutien passe d'une part par la coopération économique et commerciale: dans les pays très endettés, on appuie surtout l'industrie exportatrice, qui est considérée comme l'indispensable source de devises destinées au remboursement de la dette. D'autre part, les pays donateurs mettent en œuvre la coopération technique pour venir en aide à des programmes de développement: ils mènent des programmes régionaux, nationaux et sectoriels, et accordent aussi leur aide à d'autres projets et programmes, en vue d'améliorer les conditions de vie économiques et sociales de la population.

La crise de l'endettement exigeant des mesures d'urgence, on réserve une place toujours plus grande à la cohérence, c'est-à-dire à l'action concertée, entre la politique de développement et les politiques économique, commerciale, agraire, financière et monétaire. Il convient par ailleurs de veiller à la cohérence entre la politique de développement et les domaines qui ne relèvent pas de l'économie, tels que les politiques sociale et environnementale. Une politique de développement cohérente passe de plus en plus par une définition du cadre politique, économique, social et écologique.

* Collaboratrice du Service de désendettement de la Communauté de travail des æuvres d'entraide Swissaid/Action de carême/Pain pour le prochain/Helvetas/Caritas, Berne. 
La première partie de cet article présente l'origine de la «politique de l'endettement» et ses conséquences pour les pays subsahariens. Cette présentation se fonde sur le cas de la Tanzanie (deuxième partie), qui compte parmi les pays en développement les plus pauvres, mais aussi les plus endettés. Dans les troisième et quatrième parties, nous apprécions la cohérence entre la politique de développement de la Confédération, la politique économique et commerciale ainsi que la politique de désendettement de la Suisse en Tanzanie. En Suisse, la politique de développement relève de deux services fédéraux: le Secrétariat d'Etat à l'économie (Seco) est responsable des programmes ayant trait à la politique économique et commerciale, y compris les mesures de désendettement; la Direction du développement et de la coopération (DDC) dirige et coordonne les programmes de la coopération technique.

Voici les questions que soulève l'appréciation de la cohérence entre les activités de la Suisse en Tanzanie: les mesures appliquées par le Seco en Tanzanie ne font-elles que favoriser les exportations ou permettent-elles aussi d'améliorer la situation sociale et économique ? Ces mesures concordent-elles avec la coopération au développement menée par la DDC? Des synergies sont-elles possibles? La DDC et le Seco contribuent-ils de manière cohérente au désendettement de la Tanzanie?

\section{$\square$ Les origines de la crise de la dette dans l'Afrique du sud du Sahara}

Les crédits sont nécessaires au bon fonctionnement d'une économie car les investissements et le développement économique reposent sur la mobilisation d'un volume suffisant de ressources financières. Cependant, lorsque la dette est trop élevée en regard du potentiel économique global du débiteur, l'endettement va freiner le développement de l'économie nationale. Le rapport contractuel entre créancier et débiteur se transforme alors en un rapport de dépendance, tel que le vivent aujourd'hui la plupart des pays fortement endettés. Or, ni le créancier ni le débiteur n'est seul en cause, car tous deux peuvent être à l'origine de la crise (par manque de prudence lors de l'octroi ou de l'acceptation de crédits).

Lors de la récente crise financière, on n'a guère parlé de l'Afrique du sud du Sahara. Les flux de capitaux internationaux évitent en effet les pays africains car l'Afrique ne possède ni les infrastructures, ni la stabilité politique, ni le système bancaire et boursier nécessaires pour attirer les investissements. Il n'en reste pas moins que les pays de la région comptent parmi les plus endettés, mais aussi parmi les plus pauvres du monde. L'accumulation de leurs dettes est intervenue en deux temps: pendant la forte hausse qu'a connue l'offre de crédits internationaux privés jusqu'au début des années 80 ; puis lors du remplacement des créanciers privés par des créanciers publics'.

Ces mécanismes externes sont également pour beaucoup dans la crise de la dette au sud du Sahara. Dans les années 70, la région a en effet profité de la hausse des crédits bancaires accordés aux pays en développement, bien que le phénomène fût moins marqué en Afrique qu'en Amérique latine. A l'époque, les taux d'intérêt ont atteint des records à la basse, descendant parfois en dessous du taux de l'inflation. Ce mouvement a été provoqué par une récession dans les pays industrialisés, récession qui a conduit à une chute spectaculaire

1. CNUCED, Rapport sur le commerce et le développement 1998, Genève, 1998, p. 119. 
des investissements. Ne trouvant plus à placer leurs crédits sur les marchés intérieurs, les banques ont dû faire face à un excédent de liquidités, et suite à la crise pétrolière de 1974 l'argent coulait à flots. Cherchant des débouchés pour leurs crédits, les banques n'ont pas hésité à brader leurs prêts à des conditions défiant toute concurrence. Par ailleurs, aussi bien au Sud qu'au Nord, on pensait que les pays en développement allaient se lancer rapidement sur la voie d'une industrialisation à l'image de celle des pays occidentaux. Or, de l'avis de tous, seuls les crédits pouvaient préfinancer cette évolution et les pays en développement se sont mis à emprunter à tour de bras. Si ces prévisions s'étaient avérées exactes, l'industrialisation aurait permis de rembourser les dettes contractées. Or, au début des années 80 , les marchés financiers se sont soudain contractés: les taux d'intérêt ont explosé pour dépasser $10 \%$ et le cours du dollar a grimpé, alors que les prix des matières premières - surtout ceux des produits agricoles, qui sont la principale source de revenus des pays subsahariens - entamaient une forte baisse. Nombre de pays débiteurs se sont ainsi retrouvés dans l'impossibilité de faire face à leurs créances².

La plupart des pays africains n'étaient pas en mesure d'assumer des chocs externes et les facteurs internes suivants ont encore contribué à accroître leur surendettement: la forte hausse des emprunts à l'étranger a coïncidé avec une forte baisse des revenus des investissements à l'intérieur des pays, peut-être parce que les revenus des exportations avaient diminué ou que les années de haute conjoncture n'avaient pas été suffisamment mises à profit. Outre ces facteurs financiers, il faut mentionner un système de subvention complexe, des déficits budgétaires et des taux d'inflation élevés, l'interventionnisme de l'Etat et les obstacles commerciaux. De plus, les économies africaines souffraient de graves lacunes structurelles, parfois héritées de l'ère coloniale (la prépondérance du secteur agricole, un secteur industriel faible, une évolution structurelle insuffisants, des déséquilibres macroéconomiques, etc.). En Afrique, les classes dirigeantes n'avaient aucun intérêt à promouvoir de véritables changements. Elles profitaient de l'afflux de capitaux étrangers et mettaient la politique économique de leur pays au service de leurs propres intérêts.

Même le retrait des banques et des créanciers privés, au milieu des années 80, n'a pas suffi pour rompre le cercle infernal de la dette africaine, car l'on a alors assisté à l'arrivée en force de crédits publics, sous forme d'aide bilatérale et multilatérale, ou à titre d'octroi de garanties publiques pour les crédits à l'exportation. Depuis le début des années 90 , la place qu'occupent les créances multilatérales dans la structure de la dette ${ }^{3}$ s'est nettement accrue et elle pose un problème croissant. Jusqu'en 1996, ces créances étaient en effet exclues des opérations de désendettement et, aujourd'hui encore, seule une partie de ces créances peut être rachetée. Les créanciers des pays industrialisés du Nord et les institutions financières internationales (Banque mondiale et Fonds monétaire international notamment) portent une grande part de responsabilité dans le surendettement des pays en développement, mais ils n'ont jusqu'ici réagi qu'à court terme et trop lentement face aux problèmes de la dette africaine ${ }^{4}$.

2. Eberlein, Falk et al., Schuldenreport 1997, Bonn, 1997, p. 2 (Peter Wahl).

3. Les dettes multilatérales sont celles qui ont été contractées auprès des institutions financières internationales: Fonds monétaire international, Banque mondiale et banques de développement régionales.

4. Voir Eberlein, Falk et al., Schuldenreport 1999, Bonn, 1999, p. 24 (Reiner Falk). 


\section{$\square$ Des problèmes de liquidités aux ajustements structurels}

L'accroissement de la dette ne pouvait que renforcer la dépendance du débiteur à l'égard du créancier. Dès la fin des années 80 , les créanciers internationaux ont compris que la crise de l'endettement n'était plus un problème de liquidités conjoncturel et de courte durée, mais un véritable problème structurel. Le Fonds monétaire international (FMI) et la Banque mondiale se sont alors imposés comme gestionnaires de la crise mondiale et ont élaboré la formule du «consensus de Washington». Selon cette formule, la bonne gestion de l'économie d'un pays est liée à certaines conditions: libéralisation du commerce, stabilité macroéconomique et lutte contre l'inflation, ainsi que privatisation. On pensait que si les gouvernements remplissaient ces conditions, les marchés privés libres se chargeraient de répartir efficacement les facteurs de production et de garantir une croissance suffisante, et que cette croissance viendrait à bout des problèmes sociaux.

Pour obtenir de nouveaux crédits, la plupart des pays débiteurs sont depuis lors obligés d'appliquer des programmes d'ajustement structurels négociés avec le FMI. Les résultats de ces programmes sont néanmoins très controversés, en raison de leurs «effets secondaires» sur les groupes sociaux les plus pauvres. En Afrique notamment, les efforts d'ajustement ont engendré une hausse du produit intérieur brut (PIB) trop faible ( 2 à $3 \%$ en moyenne) pour que celle-ci bénéficie aussi aux populations démunies. Jeffrey Katz, un économiste américain, a luimême émis un constat critique en 1998: «Les programmes d'ajustement structurel du FMI et de la Banque mondiale ont poussé nombre de pays à s'endetter lourdement. Les pays qui appliquent ces programmes enregistrent une productivité si faible qu'ils ne sont tout simplement plus en mesure de rembourser la majeure partie de leur dette. $\aleph^{5}$

Depuis lors, un vif débat s'est installé sur un «consensus post-Washington», débat animé par Joseph Stiglitz, économiste en chef à la Banque mondiale jusqu'à la fin de 1999. Ce débat est sous-tendu par le double constat suivant: un Etat faible peut mettre en danger le fonctionnement du secteur privé dans le contexte mondial, et seules des institutions étatiques solides sont à même de garantir les flux internationaux de marchandises et de capitaux. Il s'agit en fait de «se doter d'un plus grand nombre d'instruments et de se fixer des objectifs plus larges», tels que le développement durable, le développement social (incluant aussi les groupes les plus démunis de la population) et le développement démocratique ${ }^{6}$. Lors de leur assemblée annuelle en automne 1999, le FMI et la Banque mondiale ont même parlé d'un changement radical et ont déclaré pour la première fois que l'ajustement structurel ne doit pas s'attacher aux seuls aspects économiques, qu'il doit prévoir aussi des mesures de lutte contre la pauvreté et consacrer plus de moyens financiers aux secteurs sociaux.

Dans le cadre de l'Initiative en faveur des pays pauvres très endettés (PPTE), les gouvernements des pays en développement élaborent désormais eux-mêmes, en collaboration avec la société civile, leurs stratégies d'ajustement et de la lutte contre la pauvreté. Le FMI et la Banque mondiale comparent ensuite ces stratégies avec leurs propres analyses et fixent la remise de dette qui sera accordée au

5. J.D. Katz, External Debt, Structural Adjustment and Economic Growth, vol. IC, UNCTAD, New York/Geneva, 1998, p. 53.

6. J. Stiglitz, More Instruments and Broader Goals: Moving towards the Post-Washington Consensus, Banque mondiale, 7.1.98. 
pays concerné. Cette nouvelle tâche constituera un nouveau défi pour la coopération internationale au développement et les bailleurs de fonds devraient continuer à privilégier la coordination de leurs programmes avec ceux d'autres partenaires.

\section{LE CAS DE LA TANZANIE}

\section{$\square$ Le développement en Tanzanie}

La dette extérieure de la Tanzanie se monte aujourd'hui à 7,9 milliards de dollars américains et ses causes relèvent en bonne partie des facteurs évoqués cidessus. Parmi les causes externes de la crise, c'est-à-dire les éléments sur lesquels la Tanzanie n'a pas ou peu de prise, relevons la deuxième hausse des prix du pétrole en 1979 et en 1980, ainsi que la chute des prix de ses principales exportations (café, thé, coton et arachide) à la fin des années 70 et au début des années 80 , baisse qui a coïncidé avec une hausse des prix à l'importation. A ces facteurs il convient d'ajouter des conditions météorologiques peu propice à la production agricole, la guerre contre l'ancien président ougandais Idi Amin Dada, les flux de réfugiés du Mozambique et, plus tard, du Rwanda, du Burundi et de la République démocratique du Congo, ainsi que la diminution générale de l'aide au développement depuis le début des années 90.

Les causes internes de la crise comprennent la stratégie de développement choisie par le pays après son indépendance. En 1967, le président Nyerere a jeté les bases d'un développement socialiste, dans lequel l'Etat exerce le contrôle suprême sur tous les secteurs. Ce faisant, il a toutefois omis de modifier le cadre économique général, peu propice à la concurrence et à l'efficacité. La grave corruption qui caractérise le pays constitue aussi un frein au développement.

\section{$\square$ Structure de la dette extérieure tanzanienne}

En 1999, le réseau Tanzania Coalition on Debt and Development (TCDD) a commandé une étude pour identifier les causes de l'endettement de la Tanzanie et proposer des solutions ${ }^{7}$. Cette étude montre que malgré la remise et le rééchelonnement de crédits publics bilatéraux (crédits de l'aide publique au développement et crédits accordés par des gouvernements), l'endettement de la Tanzanie a atteint en mars 1999 la valeur nominale de 7,9 milliards de dollars. La structure des crédits a cependant nettement évolué avec le temps : les créanciers bilatéraux ont de plus en plus accordé leur aide au développement sous forme de dons. A l'opposé, depuis le début des années 80, la part de l'endettement multilatéral (crédits accordés par la Banque mondiale, par le FMI et par la Banque africaine de développement) a nettement augmenté. Rappelons que le service des crédits multilatéraux doit être assuré sans retard pour éviter les arriérés. Le graphique suivant présente la structure de la dette tanzanienne en mars 1999:

7. L'étude, intitulée Tanzania Debt Profile, a été financée en 1996 par le projet Evangelisches Forum Schuldenerlass et menée au nom de la Tanzania Coalition on Debt and Development (TCDD). 


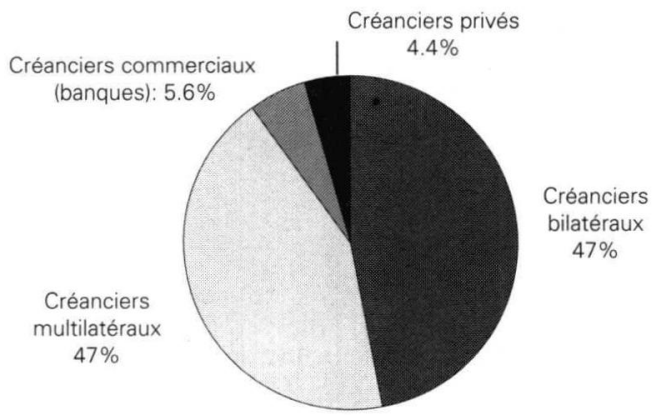

Source: TCDD, Tanzania Debt Profile, p. 2.

La majeure partie des dettes que la Tanzanie a accumulées ces dix dernières années résulte de retards de versements. Faute de devises et de recettes fiscales suffisantes, le pays n'a en effet pas pu s'acquitter de ses obligations au titre du service de la dette. Les crédits vraiment nouveaux, destinés à financer des importations et des investissements, n'ont ainsi occupé qu'une petite place au cours de cette période. En mars 1999, les arriérés de la Tanzanie totalisaient 2,6 milliards de dollars et $75 \%$ de ces arriérés concernaient des créanciers bilatéraux ${ }^{8}$.

Les éléments suivants montrent pourquoi la Tanzanie n'est pas en mesure de supporter le fardeau de sa dette:

- le montant total de la dette a augmenté de 4,5\% par an de 1986 à 1999. Dans le même temps, le produit intérieur brut n'a augmenté que de 3,5\% par an, alors que la croissance démographique atteignait $2,8 \%$;

- le service de la dette versé en 1997 et en 1998 représentait environ $17 \%$ des exportations de biens et $22 \%$ des recettes publiques. Pendant la période allant de 1993 à 1998, la Tanzanie ne s'est acquittée du service de la dette qu'à raison de $49 \%$ en moyenne.

\section{$\square$ Ajustement structurel en Tanzanie}

Pour accéder à de nouveaux crédits, le gouvernement tanzanien a mis en œuvre, dès 1986, un programme d'ajustement structurel du FMI. Ce programme comprenait diverses mesures telles que la privatisation d'entreprises publiques inefficaces, la libéralisation du système bancaire, etc. Il a toutefois dû être interrompu entre 1993 et 1995 car le gouvernement du président Mkapa était impliqué dans des affaires de corruption et les bailleurs de fonds avaient dès lors gelé leur aide à la balance des paiements et l'octroi de nouveaux crédits.

En 1995, le président en exercice, Benjamin Mkapa, a gagné les premières élections démocratiques. Pour restaurer la confiance, le nouveau gouvernement a entrepris de profondes réformes structurelles: réformes fiscales, réorganisation de l'administration des douanes, privatisation de la banque nationale, réforme du secteur social et de l'administration. Depuis 1999, l'économie tanzanienne se fonde à nouveau sur les lois du marché, l'inflation est jugulée et la balance des paiements équilibrée. De plus, le pays a lancé d'autres programmes pour renforcer le cadre institutionnel. Si la Tanzanie continue de remplir les exigences du

8. TCDD, Tanzania Debt Profile, op. cit., p. 2. 
FMI en matière d'ajustement, elle peut espérer bénéficier, en l'an 2000, d'une remise partielle de sa dette multilatérale. Reste que même une remise partielle ne réglera pas vraiment le problème de la dette tanzanienne.

\section{$\square$ L'endettement et ses conséquences sociales}

Malgré les progrès de l'économie nationale, la situation socio-économique de la majorité de la population ne s'est guère améliorée. Dans son Rapport sur le développement dans le monde pour 1998, la Banque mondiale classe ainsi la Tanzanie au sixième rang des Etats les plus pauvres. Les dépenses sociales du pays représentent entre 4 et $6 \%$ du produit national brut, alors que le fardeau élevé de la dette aggrave la précarité dans le domaine social. Une étude réalisé par Oxfam International ${ }^{9}$ a démontré que la Tanzanie dépense neuf fois plus pour le service de la dette que pour les soins médicaux de base et quatre fois plus que pour l'éducation de base. La réduction des dépenses publiques opérée dans le cadre du programme d'ajustement du FMI a eu des répercussions négatives notamment dans le secteur social. Mais c'est surtout le service de la dette multilatérale qui pose problème, car la Tanzanie doit s'acquitter de ces versements aux échéances prévues et ces versements exercent ainsi une forte pression sur les maigres revenus du gouvernement et sur le secteur social. Diverses études de la Banque mondiale font les constats suivants ${ }^{10}$ : la majorité des quelque 30 millions d'habitants vivent en dessous du seuil de pauvreté; un enfant sur sept meurt avant l'âge de 5 ans; seule la moitié des femmes ont terminé leur scolarité; le taux d'alphabétisation est passé de $85 \%$ dans les années 70 et 80 à $68 \%$ dans les années 90 ; le taux de scolarisation a également diminué pour passer de $93 \%$ en 1980 à $65 \%$ en 1995 ; le pays compte en moyenne un lit d'hôpital pour 10'000 habitants et un médecin pour 33'300 habitants; $30 \%$ de la population vivent à plus de 5 kilomètres du dispensaire le plus proche; l'espérance de vie à la naissance est de 52 ans, soit de dix ans inférieure à la moyenne d'autres pays en développement.

La conclusion saute aux yeux : il est urgent de réduire le service de la dette de la Tanzanie pour lui permettre de consacrer l'argent ainsi libéré au secteur social.

\section{LA COHÉRENCE DE LA POLITIQUE DE DÉVELOPPEMENT SUISSE EN TANZANIE}

La présente partie est consacrée à la coopération suisse au développement et à ses acteurs en Tanzanie. Sachant que le fardeau de la dette de ce pays est insupportable, les questions déjà posées dans l'introduction se font plus pressantes. A savoir : comment le Seco (avec ses mesures économiques et commerciales) et la DDC (avec sa coopération technique au développement) collaborent-ils pour soutenir les efforts de développement de la Tanzanie? Les mesures appliquées par le Seco ne font-elles que promouvoir les exportations ou contribuent-elles aussi à améliorer le contexte socio-économique? Ces mesures sont-elles en phase avec les programmes de coopération au développement de la DDC? Des synergies sont-elles possibles? La DDC et le Seco mènent-ils des activités cohérentes en faveur du désendettement de la Tanzanie?

9. Oxfam International, Debt Relief for Tansania: An Opportunity for a Better Future, avril 1998.

10. Banque mondiale, Rapport sur le développement dans le monde 1998. 
La coopération suisse au développement a pour objectif «d'améliorer les conditions de vie des habitants des pays les plus pauvres et de permettre à ces habitants de jeter les bases d'un développement durable, c'est-à-dire un développement qui ménage l'environnement, qui soit socialement supportable et qui respecte les droits de l'homme ${ }^{11}$.

La coopération au développement fait partie intégrante de la politique suisse à l'égard des pays en développement. Deux services de la Confédération sont responsables de sa conception et de sa mise en œuvre: la DDC au sein du Département fédéral des affaires étrangères (DFAE) et le Seco au sein du Département fédéral de l'économie (DFE). Comme dans nombre d'autres pays en développement, des œuvres d'entraide privées suisses mènent également des activités en Tanzanie; il s'agit aussi bien d'organisations religieuses que d'organisations non gouvernementales laïques. Les œuvres d'entraide privées collaborent étroitement avec les services de la Confédération.

La DDC dirige toutes les activités qui relèvent de la coopération au développement. Elle prend en charge les aspects opérationnels de la coopération technique, de l'aide financière et de l'aide humanitaire. Le Seco est, quant à lui, compétent pour les mesures économiques et commerciales, y compris les opérations de désendettement.

\section{$\square$ Objectifs de la coopération suisse en Tanzanie}

La Suisse soutient le développement de la Tanzanie depuis le milieu des années 70 déjà. La DDC et le Seco ont élaboré ensemble le programme de coopération avec ce pays pour les années 1999 à 2003 et s'engagent, dans ce document commun, à poursuivre les mêmes objectifs et à rechercher des synergies entre leurs activités: "La Suisse soutient les efforts de la Tanzanie pour améliorer durablement la situation économique, sociale, politique et institutionnelle, dans le but d'atténuer la pauvreté, de promouvoir la croissance économique, la bonne gestion des affaires publiques ainsi que la démocratie, et de garantir un développement qui soit à la fois durable et équilibré entre hommes et femmes. ${ }^{12}$

Voici comment le programme prévoit d'obtenir des effets synergiques: «La stratégie commune de la DDC et du Seco vise à atténuer la pauvreté en améliorant le contexte dans lequel évolue l'économie privée et en soutenant les prestations dans les secteurs essentiels pour la lutte contre la pauvreté. Par sa contribution à la balance des paiements et son aide technique, le Seco augmente les sources de revenus et accroît la disponibilité de ressources pour les secteurs visés par les activités de la DDC ([...], tels le secteur social et la construction de routes). $»^{13}$

Les secteurs prioritaires sont la santé, l'infrastructure et les transports, ainsi que la promotion de l'économie. Par ailleurs, les deux programmes de base «Développement équilibré hommes-femmes» et «Décentralisation et démocratisation» font partie intégrante des activités menées en Tanzanie.

11. DDC, La coopération internationale de la Suisse, 1998.

12. DDC/Seco, Tanzania-Swiss Country Programme 1999-2003, 1999, p. 9.

13. Ibid. 


\section{Activités du Seco en Tanzanie}

Voici les objectifs poursuivis par le Seco avec l'appui du gouvernement tanzanien: améliorer la stabilité économique du pays, accroître les exportations et promouvoir le développement de l'économie privée dans les villes et dans les régions rurales, car ces mesures sont le «moteur du développement économique et de la lutte contre la pauvreté $»^{14}$. Ces mesures comprennent les éléments concrets suivants: aide à la balance des paiements, soutien technique à la Banque nationale de Tanzanie et allégement de la dette. Le Seco s'est toutefois fixé un objectif plus large: soutenir le gouvernement tanzanien dans l'élaboration d'une politique financière stratégique, qui comprend l'élaboration du budget de l'Etat et le contrôle des dépenses. Dans le même temps, des programmes de formation renforcent les capacités institutionnelles du Ministère des finances. L'appui technique en matière de gestion financière ne devrait toutefois pas se limiter aux plus hautes sphères du pouvoir, mais bénéficier à l'avenir aussi aux gouvernements locaux dans les régions décentralisées. Ces mesures visent à terme à accroître les ressources financières consacrées au secteur social et à la lutte contre la pauvreté.

La Tanzanie n'a pu bénéficier du soutien financier de la Suisse qu'après avoir conclu un accord d'ajustement structurel avec le FMI. Le Seco accorde ainsi son appui à ce pays depuis 1986, soit une année après que la Tanzanie a signé son premier accord avec l'organisme multilatéral. Les cinq premières années, le Seco a surtout fourni une aide financière pour combler un grave manque de devises. Cette aide a permis d'importer des biens essentiels pour les secteurs pharmaceutique, agricole et sanitaire. Lorsque la Tanzanie s'est vue obligée d'interrompre son programme d'ajustement de 1994 à 1995, le Seco a également gelé son soutien économique. Et c'est en 1996, lorsque le gouvernement tanzanien a signé un nouvel accord avec le FMI, que la Suisse a repris ses activités en participant, avec d'autres donateurs, au paiement des salaires des fonctionnaires de l'Etat.

En 1997, le gouvernement tanzanien ne disposant pas de moyens suffisants pour mener un programme de stabilisation et de réforme, le Seco a contribué à son financement, ce soutien étant toutefois soumis à la condition que la Tanzanie entreprenne des réformes structurelles (privatisation de la Banque nationale du commerce, par exemple). Pour assurer le succès de cette opération, le Seco a offert à la banque les services d'un gestionnaire spécialisé. En 1998, il a en outre soutenu un projet de développement du personnel du Centre international du commerce ainsi que la fondation d'une société de leasing (ULC), et formulé les premiers éléments d'un programme de gestion de la dette pour le gouvernement tanzanien. De 1988 à 1997, le Seco a ainsi consacré 42,3 millions de francs à l'équilibre de la balance des paiements tanzanienne, 2,8 millions de francs au soutien technique et 12,6 millions de francs aux mesures de désendettement. Pour la période allant de 1999 à 2001, il prévoit de fournir au gouvernement tanzanien une aide financière de l'ordre de 20 millions de francs ${ }^{15}$.

Dans le cadre du programme allant de 1999 à 2003, le Seco a défini différents critères et échéances quant à la réalisation des programmes décrits ci-dessus. En

15. Office fédéral des affaires économiques extérieures (OFAEE), Economic and Trade-related Measures in the Context of Development Co-operation with Tanzania, Bern, 1998. 
effet, l'aide économique de la Suisse étant liée au respect des exigences du FMI par la Tanzanie, le Seco a imaginé trois scénarios possibles, allant du plus favorable au pire. Le maintien de l'aide par le Seco dépendra de la réalisation de l'un de ces scénarios - c'est-à-dire de l'interruption ou non du programme d'ajustement convenu avec le FMI.

Pour résumer, on notera que les mesures économiques et commerciales appliquées par le Seco mettent certes l'accent sur la promotion des exportations, mais qu'elles visent des objectifs plus larges. En soutenant efficacement une politique financière stratégique et en améliorant la gestion financière à tous les niveaux de l'administration publique, il devrait être possible de réorienter des ressources financières suffisantes vers le secteur social et vers la lutte contre la pauvreté. Et une telle évolution ne peut avoir que des effets positifs sur les programmes de la DDC.

\section{$\square$ Les activités de la DDC en Tanzanie}

La coopération technique de la DDC en Tanzanie dépasse les activités liées à la réalisation de projets précis, car elle vise avant tout à améliorer la répartition des deniers publics dans les secteurs prioritaires énumérés ci-dessus (santé, infrastructures et transports, ainsi que développement économique). Pour garantir la durabilité, les programmes doivent, conformément au programme élaboré par la DDC et par le Seco pour la Tanzanie, «intervenir simultanément au niveau communal et régional, ainsi qu'au niveau central. Ils doivent bénéficier du soutien technique d'institutions internationales et suisses, d'ONG et de consultants. La stratégie prévoit de passer progressivement de la réalisation de projets isolés à une approche sectorielle globale. L'équipe opérationnelle s'attachera à promouvoir activement l'apprentissage mutuel, les échanges intersectoriels, l'expérience du terrain à différents niveaux, la coordination, la création de réseaux et le dialogue politique. La DDC et le Seco soutiendront ensemble le budget global et les budgets sectoriels du gouvernement tanzanien ${ }^{16}$.

Le budget de la DDC pour les années 1999 à 2003 se situera entre 16 et 20 millions de francs suisses.

\section{Appui au secteur de la santé}

La DDC soutient la réforme du système de santé tanzanien afin d'assurer une administration efficace de ce domaine, tant au niveau national que régional. Il s'agit en fait d'intégrer les projets existants dans une approche sectorielle globale pour mieux faire correspondre les prestations aux besoins de la population. Voici comment les mesures mises en œuvre tiennent compte des exigences en matière de décentralisation spécifiées dans le programme pour la Tanzanie: le système de santé publique est géré par les services régionaux et chaque district dispose de son propre système de santé qui est intégré au programme de santé national. Le développement équilibré hommes-femmes, expressément mentionné dans le programme de coopération, occupe aussi une place importante. Les administrations locales ont en effet leur mot à dire dans la planification et dans la conception des programmes et de la gestion des prestations médicales, et elles tiennent particulièrement compte des besoins des femmes. Pour garantir l'efficacité du programme, la DDC travaille en étroite collaboration avec divers ministères et avec d'autres donateurs. Mais ce programme ne pourra remporter 
un succès à long terme que si le gouvernement tanzanien alloue plus de moyens financiers au secteur de la santé dans son budget national.

\section{Soutien au secteur infrastructures et transports}

Depuis le début des années 80 déjà, la DDC accorde son soutien au développement et à l'entretien du réseau routier dans les zones rurales. En 1991, ces activités se sont muées en un "projet routier intégré» financé par plusieurs donateurs. La coordination est assurée par le Ministère du travail et le projet a pour objectif non seulement d'étendre le réseau routier, mais aussi d'accroître les possibilités de formation pour les fonctionnaires responsables. Le programme par pays de 1999 à 2003 a également pour objectif d'améliorer la gestion du réseau routier et de renforcer les administrations locales. Les activités dans ce domaine se concentreront surtout sur la région de Morogoro.

\section{LA SUISSE ET LE DÉSENDETTEMENT DE LA TANZANIE}

\section{$\square$ Le programme suisse de désendettement}

En 1993, le gouvernement suisse concluait avec la Tanzanie un accord bilatéral de «conversion de la dette au profit du développement» et contribuait ainsi de manière notable au désendettement de ce pays. Cette opération a pu voir le jour grâce à la campagne en faveur du désendettement mise sur pied dans les années 90 par les œuvres d'entraide et les ONG. Menée sous le slogan Le désendettement: une question de survie!, cette campagne préconisait que les responsables de la crise se devaient aussi de prendre part au désendettement puisqu'ils avaient tiré profit de l'accumulation des dettes. Par ailleurs, le désendettement devait bénéficier à ceux qui souffraient le plus de la crise, soit la majeure partie des populations des pays en développement.

Entre 1989 et 1990, la pétition en faveur du désendettement a recueilli 250'000 signatures - du jamais-vu! En mai 1991, à l'occasion du $700^{\mathrm{e}}$ anniversaire de la Confédération, l'Assemblée fédérale a ainsi fini par débloquer 400 millions de francs suisses pour un programme de désendettement (auquel étaient également alloués 100 millions de francs provenant d'un crédit précédent) et 300 millions de francs pour financer des projets destinés à préserver l'environnement mondial. Sur les 500 millions de francs destinés aux actions de désendettement, 400 millions ont jusqu'ici financé les opérations suivantes: rachat et remise de dettes au bénéfice d'une garantie publique, participation à des rachats coordonnés au niveau international, rachat de dettes commerciales auprès de banques privées, participation au financement d'arriérés auprès d'institutions financières multilatérales et soutien à un programme de gestion de la dette assisté par ordinateur. Ce logiciel informatique revêt de plus en plus d'importance car il facilite la gestion de la dette et la rend transparente. Nous décrivons ci-après les mesures qui ont été prises pour réduire la dette tanzanienne.

\section{$\square$ Programme de rachat de dettes commerciales}

Dans le cadre du programme suisse de désendettement, le Seco a versé au milieu de 1999 un montant de 10 millions de francs suisses à un programme de désendettement international ${ }^{17}$ destiné à réduire la dette commerciale de la

17. Programme de l'Association internationale de développement (AID) pour le rachat de dettes commerciales; voir DDC/Seco, Tanzania-Swiss Country Programme 1999-2003, op. cit. 
Tanzanie. Les dettes pouvant bénéficier d'une remise dans le cadre de cette opération se montent au total à 632,2 millions de dollars. Elles comprennent des montants dus à des fournisseurs et à des banques commerciales ainsi que des dettes contractées auprès de sociétés de télécommunication et auprès d'autres entreprises, la majeure partie de ces dettes ayant été accumulée entre la fin des années 70 et le milieu des années 80 .

Cette opération de désendettement permet au gouvernement tanzanien de racheter ses dettes contre de l'argent comptant, le taux de conversion étant de 1 dollar de dettes pour 12 cents. Le montant converti comprend les crédits de plus de 1000 créanciers. Cette opération est vitale car les crédits commerciaux n'affluent plus vers les pays du Sud comme ce fut le cas dans les années 70. Pire encore, la Tanzanie n'est plus considérée comme un pays solvable au niveau international, car les investisseurs ne veulent plus prendre de risque, et cette situation entrave le développement du pays. L'opération de désendettement offre à présent à la Tanzanie l'occasion de s'acquitter de son propre chef du service de ces dettes commerciales et de redorer son blason sur les marchés financiers internationaux. Elle pourra ainsi améliorer ses relations avec les créanciers et obtenir de nouveaux crédits pour promouvoir l'économie privée et attirer des investisseurs étrangers. Pour éviter que la dette ne suive à nouveau une courbe exponentielle, les nouveaux crédits sont liés à l'application des programmes d'ajustement structurel mentionnés plus haut.

\section{$\square$ Participation du Seco à des opérations de désendettement multilatérales}

La Suisse a participé à raison de 40 millions de francs à l'Initiative en faveur des pays pauvres très endettés (PPTE) lancée par la Banque mondiale et le FMI. Grâce à cette initiative, la Tanzanie devrait obtenir une remise partielle de sa dette extérieure à la fin de l'an 2000.

Comme nombre de pays pauvres, la Tanzanie n'avait pas mis en place une gestion de la dette transparente et à long terme. La Suisse a dès lors collaboré avec l'Autriche, la Suède et le Danemark pour doter ce pays d'un système de gestion efficace et pour former les fonctionnaires chargés de cette gestion.

\section{$\square$ Les fonds de contrepartie suisses}

La campagne en faveur du désendettement de 1989-1990 exigeait notamment que les moyens financiers libérés par la remise de dettes au niveau macroéconomique bénéficient directement à la population pauvre. La communauté internationale s'est dès lors vivement intéressée à la notion de «désendettement créatif »: les gouvernements bénéficiant de la remise d'anciens crédits à l'exportation doivent reverser une partie du montant économisé converti en monnaie locale dans un fonds dit de contrepartie. L'argent sert à financer des projets de lutte contre la pauvreté, et la société civile (ONG, associations d'entraide, etc.) du pays concerné doit prendre part à une partie des décisions concernant l'utilisation de cet argent. Or, dans le cadre de tous les fonds de contrepartie suisses, les ONG sont également les bénéficiaires des projets financés par les fonds.

Depuis 1992, les dettes que 19 pays ont contractées auprès du gouvernement suisse en convertissant des crédits à l'exportation ont été annulées. Elles se montaient au total à 1,1 milliard de francs. Un fonds de contrepartie a été créé dans 12 de ces pays. Le programme suisse de fonds de contrepartie repose sur 
une collaboration efficace entre le Seco, la DDC et le Service du désendettement de la Communauté de travail des œuvres d'entraide Swissaid/Action de carême/Pain pour le prochain/Helvetas/Caritas. Le Seco est responsable de tous les fonds de contrepartie. Il négocie les accords de désendettement avec les gouvernements concernés, ainsi que le montant de contrepartie. Il arrive que ces accords fixent déjà la stratégie de financement, la durée et l'orientation du fonds de contrepartie. La DDC est responsable du suivi opérationnel du fonds, tandis que le Service du désendettement fait office de consultant auprès du Seco et de la DDC, et collabore à la conception, à la création et au suivi des fonds de contrepartie. Les représentations suisses dans les pays endettés jouent parfois un rôle important au sein des organes décisionnels des fonds de contrepartie.

\section{$\square$ Le Trust Fund Tanzanie-Suisse}

En 1993, la Suisse a aussi conclu avec la Tanzanie un accord prévoyant la remise de 33 millions de francs suisses de dette. Dans cet accord, le gouvernement tanzanien s'engageait à verser $15 \%$ de cette somme (soit 3,4 millions de dollars) à titre de contrepartie sur un fonds. Grâce au versement des intérêts, ce fonds totalisait 5,5 millions de dollars à mi-août 1999. De ce total, $40 \%$ peuvent servir à financer des projets de développement menés par des ONG et par des organismes d'entraide locaux, ainsi que par des organisations privées, ou à soutenir les programmes mis en œuvre par le gouvernement lui-même. Les $60 \%$ restants devraient alimenter les caisses de l'Etat au titre d'aide à la balance des paiements, ce qui n'a toutefois pas été le cas jusqu'ici.

Le Trust Fund Tanzanie-Suisse est géré par un conseil de trois membres, qui comprend un représentant du Ministère des finances, un représentant du Ministère pour l'administration régionale et le responsable du bureau de coordination de la DDC en Tanzanie. Une secrétaire générale est chargée de régler les affaires courantes du Trust Fund ainsi que d'opérer un premier tri des projets et d'assurer ensuite leur suivi. Depuis 1994, 516 projets ont été présentés, dont 86 ont été retenus jusqu'ici. Le nombre des projets rejetés montre clairement à quel point les couches de population les plus pauvres ont besoin d'un soutien financier.

Les projets acceptés et financés sont menés exclusivement par des organisations non gouvernementales, et les secteurs concernés sont les suivants: santé, lutte contre la pauvreté, développement communal et protection de l'environnement. Ces projets comprennent par exemple l'appui financier à un hôpital, à une école ou à un centre de formation, ou l'équipement d'un centre de formation professionnelle. On sélectionne volontairement les projets qui touchent les mêmes secteurs que ceux visés par le programme de la DDC, afin de garantir la cohérence entre ces deux secteurs d'activité et d'exploiter les synergies potentielles. En août 1999, tous les moyens à disposition ont été attribués de sorte que le Trust Fund sera probablement clos à la fin de l'an 2000.

\section{CONCLUSION}

Une analyse du problème de la dette basée sur le cas de la Tanzanie montre que le surendettement des pays les plus pauvres entrave leur développement économique et social. Une évolution positive à long terme de ces pays passe non seulement par la réduction de leur dette, mais aussi par la promotion de leur développement dans 
les domaines politique, économique et social. En prenant des mesures allant dans ce sens, il convient toutefois de garder à l'esprit que la responsabilité de l'endettement relève aussi bien des pays créanciers que des pays débiteurs.

L'examen du programme de coopération de la Suisse avec la Tanzanie indique que les diverses mesures de développement et de désendettement sont en principe cohérentes, puisque la DDC et le Seco s'engagent à rechercher des synergies entre les différentes activités. Cette volonté n'est pas toujours aussi manifeste dans le travail avec les autres pays de concentration de la coopération suisse au développement. L'absence de collaboration et de cohérence est souvent due à des facteurs historiques aléatoires ou aux intérêts personnels des responsables. Mais la situation diffère dans le cas de la Tanzanie, car la Suisse met, depuis le milieu des années 90, l'accent sur l'intégration et sur la coordination de ses diverses activités.

Ces principes servent aujourd'hui de modèle à une approche coordonnée et cohérente du développement, qui tient compte des besoins de l'ensemble d'un secteur. Il convient de relever en particulier que les mesures connexes appliquées par le Seco au niveau macro-économique contribuent à façonner le contexte général pour que les efforts entrepris par le gouvernement tanzanien se traduisent effectivement par des progrès sur la voie du développement. Les activités de la DDC apportent à double titre un appui à cette politique: d'une part, les moyens disponibles dans les secteurs prioritaires que sont la santé ainsi que la construction de routes et les transports atteignent une plus grande efficacité lorsque leur utilisation est décentralisée; d'autre part, les activités de la DDC favorisent la participation des populations les plus pauvres aux processus de décision et, surtout, la participation des femmes au processus de développement.

Seuls des remises de dettes et l'abaissement de la dette à un niveau supportable permettront à la Tanzanie de se développer. Dans les années 70, la Suisse a déjà annulé toutes ses créances accordées au titre de l'aide au développement. Et, ces dernières années, elle a aussi fait office de pionnier sur le plan international en plaidant en faveur de la réduction de tous les autres types de dettes, aussi bien les dettes commerciales que les crédits commerciaux au bénéfice d'une garantie bilatérale publique. La Suisse a aussi été l'un des premiers pays à contribuer financièrement à l'initiative de désendettement multilatérale (en faveur des PPTE) lancée par la Banque mondiale et par le FMI, et cette initiative bénéficiera également à la Tanzanie dans les années à venir.

En créant un Trust Fund Tanzanie-Suisse, notre pays a par ailleurs trouvé une solution «créative» au problème que posait l'affectation de l'argent libéré par les remises de dettes. Puisqu'il s'agissait d'assurer que les allégements du budget national au niveau macroéconomique bénéficient aux populations les plus pauvres, l'argent provenant de la conversion d'une petite partie de la dette est versé dans le Trust Fund. Celui-ci finance directement des projets d'organisations non gouvernementales, de sorte que l'argent vient directement en aide aux plus démunis.

Pour ce qui est de la création et de la gestion du fonds de contrepartie, il convient de souligner la cohérence entre les trois services concernés (Seco, DDC et Service du désendettement), qui mettent en commun leurs compétences stratégiques pour appliquer cet instrument. Or, parvenir à un minimum de cohérence exige une coopération active et sans préjugés, ainsi que la volonté de tirer 
les enseignements des échanges réciproques. Il faut pour cela entretenir un dialogue permanent et savoir réagir avec souplesse face à une situation qui ne cesse d'évoluer.

Les fonds de contrepartie ne sont bien sûr pas à même de remplacer une politique conséquente des pays débiteurs ni de mettre en place un développement durable dans ces pays. Ils n'en restent pas moins un instrument efficace et un exemple probant des possibilités d'investir l'argent libéré par une remise de dette dans la lutte contre la pauvreté, tout en y faisant participer la société civile. Les créances suisses ne représentant toutefois qu'une part minime de la dette extérieure des pays pauvres, les effets d'une opération de désendettement entreprise par la Suisse demeurent modestes. De plus, on ne peut pas exiger des bailleurs de fonds internationaux qu'ils contribuent à long terme à façonner la politique de développement et de désendettement des pays pauvres. Les gouvernements et les parlements de ces pays doivent en effet assumer un rôle plus actif et élaborer une véritable stratégie dans ces domaines. A l'avenir, ils devraient aussi s'attacher d'avantage à mettre en place des mécanismes de décision et d'établissement du budget plus transparents et plus efficaces, ainsi qu'à faire participer la population concernée aux prises de décision. 